\title{
A BAW Resonator Based RF Subsampling Band Pass $\Sigma \Delta$ Modulator
}

\author{
Mohamed Béchir Dadi and Ridha Bouallegue
}

\begin{abstract}
A new design technique of RF subsampling continuous time bandpass sigma delta modulator is presented in this paper. The modulator employs a Bulk Acoustic Wave (BAW) resonator. A robust method to eliminate the anti-resonance frequency of the resonator is detailed. Combining subsampling process with BAW technology offers a promising solution to reduce sampling frequency and power consummation of the $\Sigma \Delta$ modulator with high quality-factor. A fourth-order continuous time subsampling bandpass $\Sigma \Delta$ modulator is studied as an example. The Signal Transfer Function and Noise Transfer Function are compared in this context. Besides, the effects of employing BAW resonator on the output SNR of the modulator is studied. Simulation results show that using an input RF signal of $1.56 \mathrm{GHz}$, the SNR measured with and without anti-resonance cancellation are equal,respectively, to $52 d B$ and $36 d B$ when subsampled the signal with $902.85 \mathrm{MHz}$.
\end{abstract}

Index Terms-BAW resonator, RF Subsampling, bandPass Sigma Delta modulator.

\section{INTRODUCTION}

Continuous time bandpass sigma delta modulator is seen as a promising candidate for fulfill RF Analog to Digital Converter (ADC) with high speed, high resolution and low power consumption in the context of software radio receivers[1]. RF down-conversion based $\Sigma \Delta$ modulator could be performed by subsampling process in order to reduce sampling frequency. At high frequencies, Continuous-Time (CT) bandpass loop filter of the $\Sigma \Delta$ modulator is usually based on integrated LC resonators [2]. However, the low quality factor $\mathrm{Q}$ of the $\mathrm{CT}$ resonators can limit the performance of the modulator, particularly for narrowband applications [2]. A Q-enhancement technique can be employed in order resolve this problem [3]. Nevertheless, non linearity and power consumption of the modulator will be increased [4], [5]. On the other hand, it is known that electro-mechanical resonator such as MEMS (MicroElectroMechanical System), SAW (Surface Acoustice Wave) and BAW (Bulk Acoustic Bave) have a high Q-factor [6]. Thus, they can surmount the limitation of the classical resonators based on integrated LC resonators in CT bandpass $\Sigma \Delta$ modulators. MEMS and SAW resonator present high Q-factor but compatibility issues with CMOS technology and high insertion loss for some configurations will limit its performances. Contrary to MEM and SAW resonators, BAW technology have a lower insertion loss, better selectivity and

Manuscript received June 1, 2012; revised July 1, 2012.

The authors are with the Innov'Com Laboratory, Sup'Com, University of Carthage, Tunis, Tunisia (e-mail: Mohamed.dadi@isimg.rnu.tn, Ridha.bouallegue@supcom.rnu.tn). can be directly integrated above IC[7]. It can offer more opportunities to realize a RF reconfigurable filters for multi-standard receivers. In the present work, we propose to using BAW resonator in order to design a CT-bandpass loop filter for the subsampling $\Sigma \Delta$ modulator.

This paper is organized as follows: Section II reviews BAW resonator characteristics. The proposed subsampling modulator based BAW resonator is described in Section III. A design example and performances analysis of the proposed modulator are studied in this section. Some conclusions are depicted in Section IV.

\section{BAW RESONATOR: STRUCTURE AND CHARACTERISTICS}

\section{A. Basis Architecture}

Two structures are used to build a BAW resonator: FBAR (Film Bulk Acoustic Resonator) and SMR (Solidly Mounted Resonator). A FBAR resonator is a think piezoelectric layers comprised between two electrodes and the acoustic isolation is effected by air gaps. The SMR structure is identical to the first but the isolation is achieved using Bragg reflector. An example of a BAW-SMR resonator structure is shown in FIg. 1. The resonator is composed of alternative layers of low and high acoustic impedance, respectively. The number

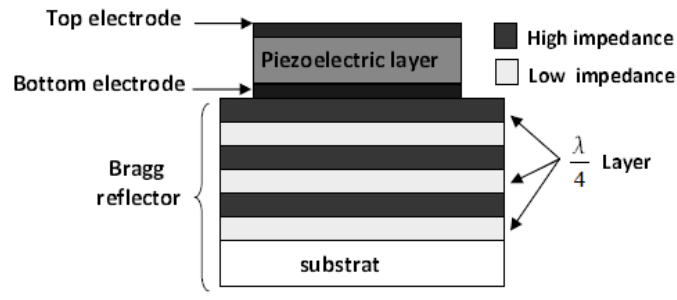

Fig. 1. BAW-SMR resonator

of layers depends on the acoustic impedance ratio between the straight layers[8].

\section{B. Butterworth Van Dyke Model}

The BAW resonator can be studied by using compact electrical model. As described in FIg. 2, the electrical circuit known as "Butterworth Van Dyke Model" can model the BAW resonator. $L_{m}$ and $C_{m}$ designed the motional behavior of piezoelectric resonator. $R_{m}$ is the mechanical losses in the resonator. $C_{p}$ is the electrostatic capacitor.

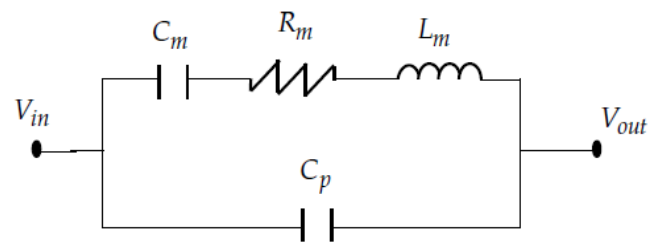

Fig. 2. BVD Model of BAW Resonator 
The transfer function of the BVD model of the BAW resonator is given by [6]:

$$
H(s)=\frac{1}{s C_{p}} \frac{1+\frac{s}{Q_{s} w_{s}}+\frac{s^{2}}{w_{s}^{2}}}{1+\frac{s}{Q_{p} w_{p}}+\frac{s^{2}}{w_{p}^{2}}}
$$

where:

$$
\begin{aligned}
w_{s} & =\frac{1}{\sqrt{L_{m} C_{m}}} \\
w_{p} & =w_{s} \sqrt{1+\frac{C_{m}}{C_{p}}} \\
Q_{s} & =\frac{1}{w_{s} R_{m} C_{m}} \\
Q_{p} & =\frac{1}{w_{p} C_{m} R_{m}}
\end{aligned}
$$

Here, $w_{s}$ and $w_{p}$ designed, respectively, the anti-resonance and resonance pulsations. $Q_{s}$ and $Q_{p}$ are, respectively, the anti-resonant and resonant quality factors. An example of the frequency response of the transfer function response of the BAW-SMR resonator is shown in FIg. 3. The resonant frequency is choosen to $f_{p}=1.58 \mathrm{GHz}$ and the anti-resonant frequency is equal to $F_{s}=1.42 \mathrm{GHz}$. Where $L_{m}=20.6 \mathrm{nH}$, $C m=0.61 p H, R_{m}=0.26 \Omega$ and $\left.C_{p}=2.54 p H\right)$. In this example the quality factor $Q_{s}$ and $Q_{p}$ are equal respectively to 707 and 635. It is clear that an anti-resonant notch is produced.

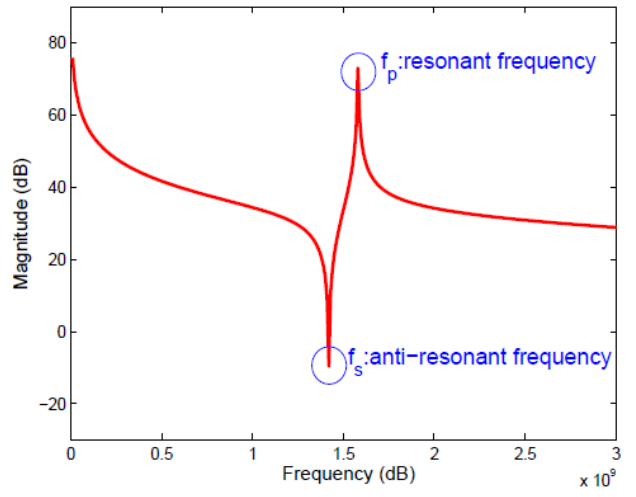

Fig. 3. Frequency response of the BAW resonator

in the magnitude response of the transfer function, $H(s)$. This problem is due to the parasitic capacitance $C_{p}$. It can deteriorate the filter response of the modulator around the central frequency. Consequently, performances of the modulator will be degraded significantly.

\section{Anti-Resonance Cancellation Technique}

In order to cancel the effect of this anti-resonance, a negative capacitance $C_{c}$ is used as following in FIg. 4 .

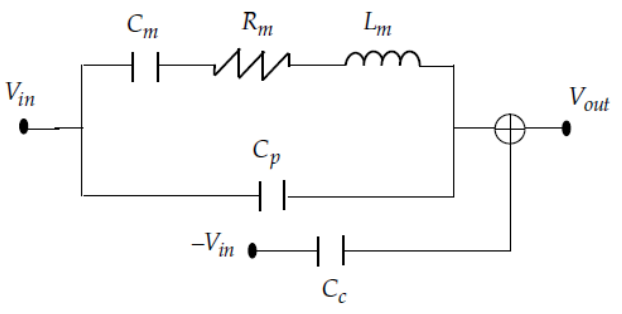

Fig. 4. Anti-resonance cancellation circuit
Then, the modified transfer function of the BAW resonator can be written as[6]:

$$
H_{\text {cancel }}(s)=H(s)-\frac{1}{s C_{c}}
$$

Using equation (1),we get:

$$
H_{\text {cancel }}(s)=\frac{s\left(C_{m} / C_{p}^{2}\right)}{s^{2}+\left(\frac{w_{p}}{Q_{p}}\right) s+w_{p}^{2}}
$$

As shown in FIg. 5, if the value of $C_{c}$ is equal to $C_{p}$, the effect of $C_{c}$ will be completely canceled. Consequently, the

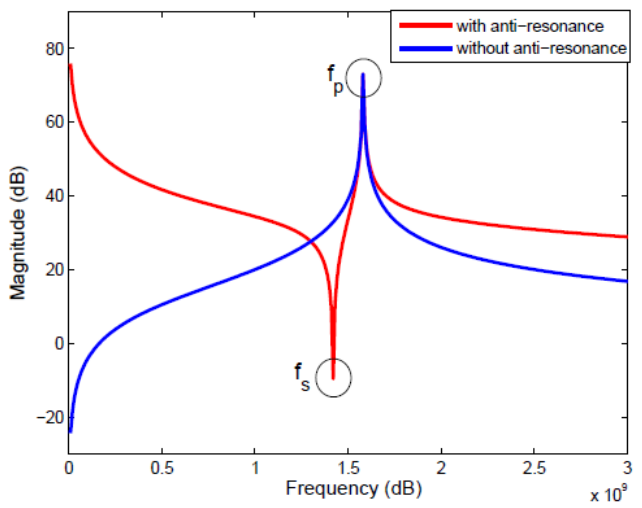

Fig. 5. BAW resonator transfer function with and without antiresonance

anti-resonance component is canceled and the equation (4) is similar to the transfer function of a $\mathrm{LC}$ resonator with a quality factor $Q_{p}$ equal to 635. Finally, a BAW compensated resonator with high quality factor has been obtained. It can be used as a continuous time loop filter for bandpass $\Sigma \Delta$ modulator.

\section{SUBSAMPLING CONTINUOUS BANDPASS $\Sigma \Delta$ MODULATOR BASED BAW RESONATOR}

The As detailed in [9], a subsampling continuous bandpass $\Sigma \Delta$ modulator is the best candidate to downconvert a RF bandpass signal with lower sampling frequency and lower power consumption. In this section, a BAW resonator based CT-bandpass loop filter is used in order to design a fourth order subsampling $\Sigma \Delta$ modulator.

\section{A. The Proposed $\Sigma \Delta$ Modulator}

An example of a subsampling bandpass CT $-\Sigma \Delta$ Modulator with BAW resonator is shown in FIg. 6. The loop filter of

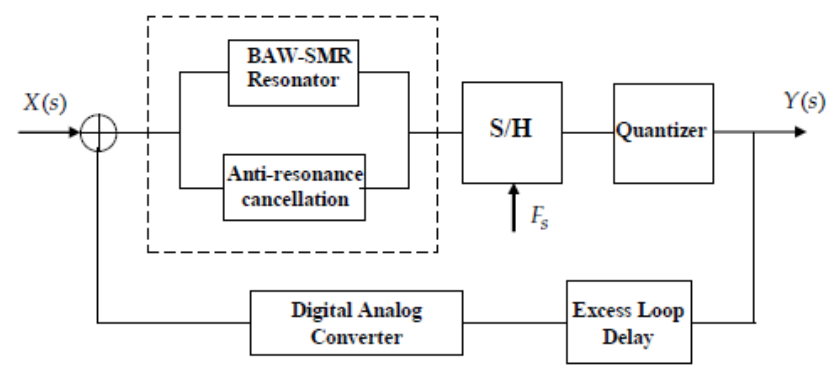

Fig. 6. Block diagram of a CT subsampling $\Sigma \Delta$ modulator

the $\mathrm{CT}$ proposed modulator is based on BAW resonator. The problem of anti-resonance is cancelled by adding negative capacitance circuit as detailed in the previous section. A 
Sample and Hold (S/H) block is used in order to subsampling the signal at the output of the resonator with a sampling frequency $F_{s}$. As detailed in [9], the relation between $F s$ and the center frequency $f_{p}$ is given by:

$$
F_{S}=\frac{4 f_{p}}{2 N+1}, N>0
$$

Here $N$ is the subsampling ratio.

The excess loop delay in the proposed modulator is designed by $e^{-\alpha / F s}$, where $\alpha$ designed the total delay in the feedback path of the modulator. A sine shaped pulse is assumed for the DAC in order to reduce the jitter effects on the modulator. A linear model of a fourth order CT-subsampling $\Sigma \Delta$ modulator is presented in FIg. 7[9]. Where, $R_{1}$ and $R_{2}$ designed two identical second order continuous time BAW resonators with the same transfer function $H(s)$. As detailed in [9], the open

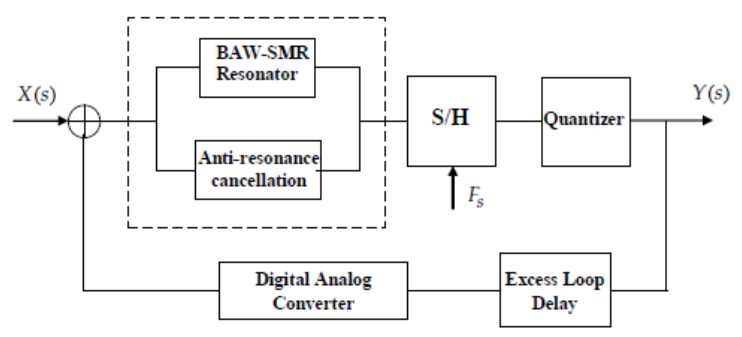

Fig. 7. A linear model of the proposed $\Sigma \Delta$ modulator

loop transfer function of the modulator in the z-domain can be written as:

$$
\left.G H(z)=Z\left[L^{-1}\left(\left(k_{1} H(s)^{2}+k_{2} H(s)\right) H_{d a c}(s) e^{-a s}\right)\right)\right]
$$

where $H_{d a c}$ is the impulse response at the output of the sine shaped DAC. It is expressed as[9]:

$$
H_{d a c}(s)=\frac{w_{d a c}{ }^{2}\left(1-e^{-s T_{e}}\right)}{s\left(s^{2}+w_{d a c}{ }^{2}\right)}
$$

Here, $w_{d a c}=2 \pi f_{d a c}$. Where $f_{d a c}$ is the input frequency of the sine shaped signal at the DAC[9]. The Signal and Noise Transfer Function respectively $\operatorname{STF}(z)$ and $\operatorname{NTF}(z)$ are given by:

$$
\begin{gathered}
\operatorname{STF}(z)=\frac{Z\left[L^{-1}\left(H(s)^{2}\right)\right]}{1+G H(z)} \\
\operatorname{NTF}(z)=\frac{1}{1+G H(z)}
\end{gathered}
$$

\section{B. Simulation Results and Performances Analysis}

Assuming that each BAW resonator based $\Sigma \Delta$ Modulator in FIg. 7 have a resonant frequency $f_{p}=1.58 \mathrm{GHz}$ and an anti-resonant frequency $F_{s}=1.42 \mathrm{GHz}$ with a undersampling ratio $N=3$. Then, the sampling frequency, $F_{s}$ is around $902.85 \mathrm{MHz}$ and the excess loop delay, $t_{d}=1.5=F_{s}$ and $f_{\text {dac }} 1.8$ $\mathrm{GHz}$. In order to preserve the stability of the modulator, the coefficients of loop filter are choosing as $k_{1}=k_{2}=0.7$. The NTF and the STF frequency response deduced from equation

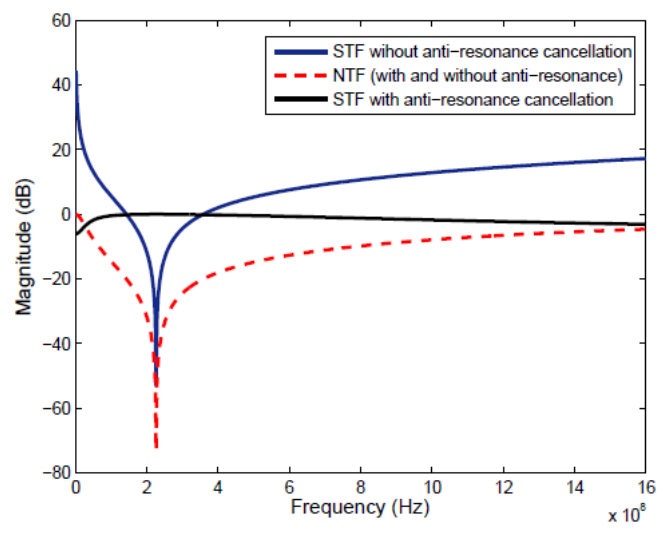

Fig. 8. STF and NTF transfer function of the proposed modulator with BAW resonator

(8) and (9) of the proposed modulator with and without anti-resonance cancellation technique are given in FIg. 8. We can see the anti-resonance effects in the STF frequency response. In fact, without the anti-resonance cancelation, an unwanted peak appears to the intermediate frequency, $f_{I F}=$ $F_{s} / 4=225.71 \mathrm{MHz}$ and STF magnitude at the output of the modulator is minimal around this frequency. Although, using a BAW with anti-resonance technique, the signal input is filtered without undesirable frequency components. While, the NTF frequency response is identical with and without anti-resonance cancellation. This problem can lead to negative effects on the SNR at the output of the modulator. In FIg. 9.

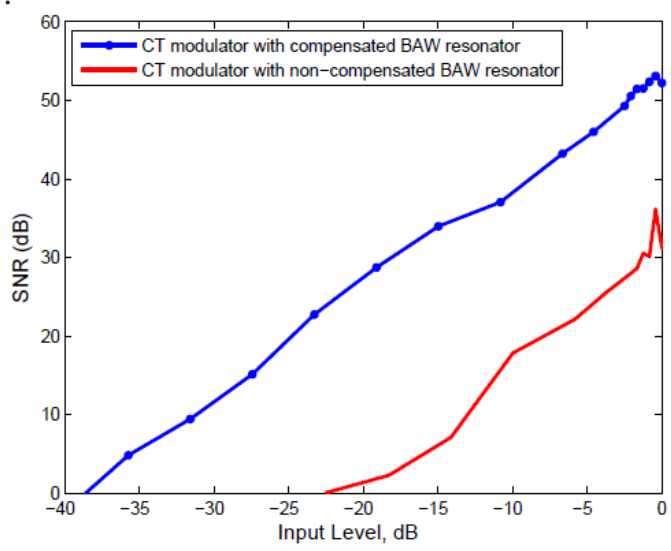

Fig. 9. SNR versus input amplitude of a CT-subsampling $\Sigma \Delta$ Modulator with and without compensated BAW resonator

We have plotted the SNR at the output of the proposed BAW modulator with and without anti-resonance system. We can see that the maximum attainable SNR with non-compensated BAW resonator (with anti-resonance) is 52 $d B$. While, if we use an anti-resonance cancellation system, the maximum value of SNR is reduced to $38 \mathrm{~dB}$. We can conclude that the using of BAW resonator based subsampling CT $-\Sigma \Delta$ modulator, the SNR will be deteriorated at the output of the modulator. However, we can resolve this problem with anti-resonance system.

\section{CONCLUSION}

In this paper, a new design technique to optimize the CTsubsampling bandpass $\Sigma \Delta$ modulator is presented. The CTloop filter of the modulator is based on BAW resonator.Antiresonance is the major issue in the kind of the resonator. A design method to cancel this problem is 
discussed. A design example for a CT-subsampling bandpass fourth order $\Sigma \Delta$ modulator based BAW resonator is simulated.

\section{REFERENCES}

[1] K. P. J. Thomas, R. S. Rana, and Y. Lian, "A 1 GHz CMOS Fourth-Order Continuous-Time Bandpass Sigma-Delta Modulator For RF receiver End A/D Conversion," in Proceedings of Asia and South Pacific Design Automation Conference, vol. 2, pp. 665-670, 2005.

[2] B. K. Thandri and J. Silva-Martinez, "A $63 \mathrm{~dB}$ SNR, 75-mW bandpass RF $\Sigma \Delta \mathrm{ADC}$ at $950 \mathrm{MHz}$ using $3.8-\mathrm{GHz}$ clock in $0.25-\mathrm{SiGe}$ BiCMOS technology," IEEE J. Solid-State Circuits, vol. 42, no. 2, pp. 269-279 and 876-880.

[3] E. Avignon et al., "Design of a $3 \mathrm{GHz}$ 6th order delta-sigma modulator in a $0.2 \mathrm{~m}$ GaAs Technology," in Proc. 4th IASTED International Conference on Circuits, Signals and Systems, pp. 205-210, San Francisco(USA), Nov. 20-22, 2006.

[4] A. N. Mohieldin, E. Snchez-Sinencio, and J. Silva-Martinez, "A Design Considerations of Bandpass LC Filters for RF Application," in Proc. International Conference on Electronics, Circuits and Systems, Cairo, Egypt, pp. 404- 409, Dec. 1994.

[5] W. Gao and W. M. Snelgrove, "A 950-MHz IF Second-Order Integrated LC Bandpass Delta-Sigma Modulator," IEEE Journal of Solid-State Circuits, vol. 33, no. 5, pp.723-732, May 1998.

[6] J. Farakh, A. Hassan, B. Nicolas, and M. Dominique, "The Design of RF Bandpass $\Sigma \Delta$ Modulators with Bulk Acoustic Wave Resonators," IEEE International Symposium on Circuits and Systems (ISCAS'2009), Taipei, Taiwan, May 2009.

[7] M. A. Dubois et al., "Above-IC Integration of BAW Resonators and Filters for Communication," Presented at 3rd Intern. Symp. on Acoustic Wave Devices for Future Mobile Communication Systems, vol. 2B-3, 2007.

[8] M. El Hassan, E. Kerherve, Y. Deval, and D. Belot, "A New Method to Reconfigure BAW-SMR Filters Using CMOS Transistors," Microwave Symposium, IEEE/MTTS International, pp. 1603-1606, June 2007
[9] M. B. Dadi and R. Bouallegue, "Subsampling Continuous Time Bandpass $\Sigma \Delta$ modulator for Radio Frequency A/D Conversion," in Proc. $10^{\text {th }}$ International Conf. on Information Science, Signal Processing and their Applications (ISSPA 2010), pp. 181-184, Mai 2010.

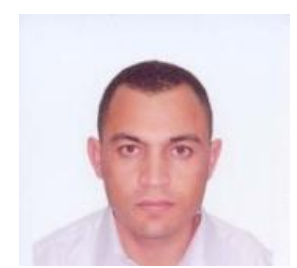

Mohamed Béchir Dadi received the M. S degree in Telecommunication in 2005 and the M.Sc. degrees telecommunication system. In Mars 2011, he also received the Ph.D degrees in telecommunication all from National Engineer School of Tunis (ENIT). Since September 2012, he was an assistant professor in the National Engineer School of Gabès (ENIG). His current research interests include Software Radio, RF subsampling, Sigma Delta Modulator and Wireless system.

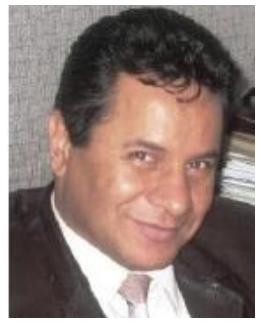

Ridha Bouallegue (M'98) received the $\mathrm{Ph}$. D degrees in electronic engineering from the National Engineering School of Tunis. In Mars 2003, he received the Hd.R degrees in multiuser detection in wireless communications. From September 1990 he was a graduate Professor in the higher school of communications of Tunis (SUP'COM), he has taught courses in communications and electronics. From 2005 to 2008, he was the Director of the National engineering school of Sousse. In 2006, he was a member of the national committee of science technology. Since 2005, he was the laboratory research in telecommunication Director's at SUP'COM. From 2005, he served as a member of the scientific committee of validation of thesis and Hd.R in the higher engineering school of Tunis. His current research interests include wireless and mobile communications, OFDM, space-time processing for wireless systems, multiuser detection, wireless multimedia communications, and CDMA systems. 